\title{
Local Structure of Ferroic Iron Formates at Low Temperature and High Pressure Studied by Mössbauer Spectroscopy
}

\section{Supporting Information}

\author{
Ines E. Collings ${ }^{1,2, *,}$, Denis M. Vasiukov ${ }^{1,3}$, Catherine A. McCammon ${ }^{3}$, Leonid Dubrovinsky ${ }^{3}$, \\ Valerio Cerantola ${ }^{2}$, Sylvain Petitgirard ${ }^{3}$, Christian B. Hübschle ${ }^{1}$, Andreas Schönleber ${ }^{1}$, \\ Dmitry Chernyshov ${ }^{2}$, Sander van Smaalen ${ }^{1}$, and Natalia Dubrovinskaia ${ }^{1}$ \\ ${ }^{1}$ Laboratory of Crystallography, University of Bayreuth, 95440 Bayreuth, Germany. \\ ${ }^{2}$ European Synchrotron Radiation Facility, 71 avenue des Martyrs, 38000 Grenoble, France. \\ ${ }^{3}$ Bayerisches Geoinstitut, University of Bayreuth, 95440 Bayreuth, Germany. \\ ${ }^{\dagger}$ Current Address: Center for X-ray Analytics, Swiss Federal Laboratories for \\ Materials Science and Technology, Überlandstrasse 129, 8600 Dübendorf, Switzerland.
}

*E-mail: ines.collings@empa.ch 


\section{Contents}

$1 \quad$ LT-MS of DMAFeF $\quad$ S3

2 Low-temperature SCXRD $\quad$ S6

3 HP-MS for AFeF $\quad$ S9

4 HP-MS for DMAFeF $\quad$ S10

$5 \quad$ LT- and HP-SMS for AFeF $\quad$ S13

6 LT- and HP-SMS for DMAFeF $\quad$ S15 


\section{LT-MS of DMAFeF}

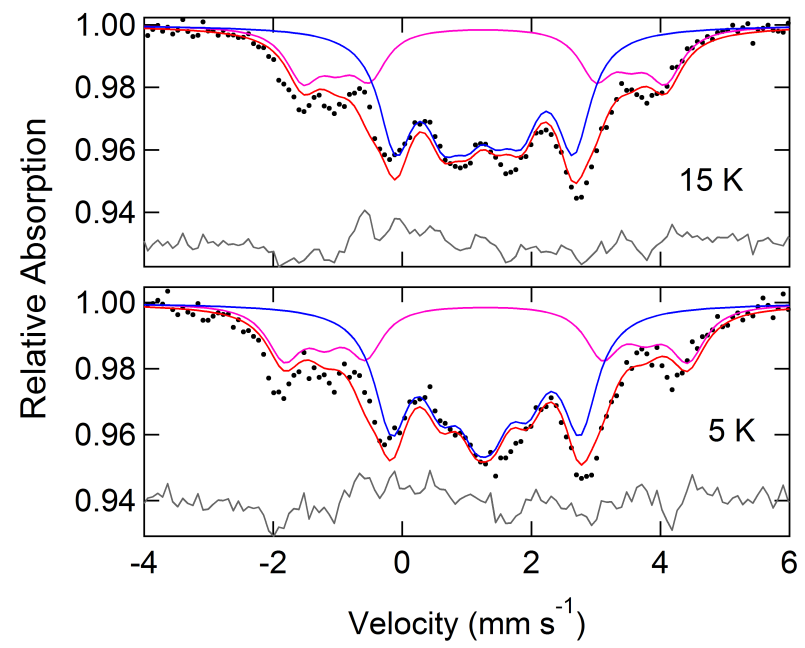

Figure S1: Magnetic sextet of DMAFeF at 15 and $5 \mathrm{~K}$ fitted with a two-site Fe model. The black points indicate the collected data, the red line is the total fit to the spectrum, the blue and pink lines are from the different Fe environments, and the grey line indicates the difference between the data and the overall fit. The IS and peak widths are constrained to be the same. The intensity ratio of the pink subspectrum is also constrained to be half of the blue subspectrum giving the 1:2 area ratio of the two subspectra as was refined at $80 \mathrm{~K}$. 


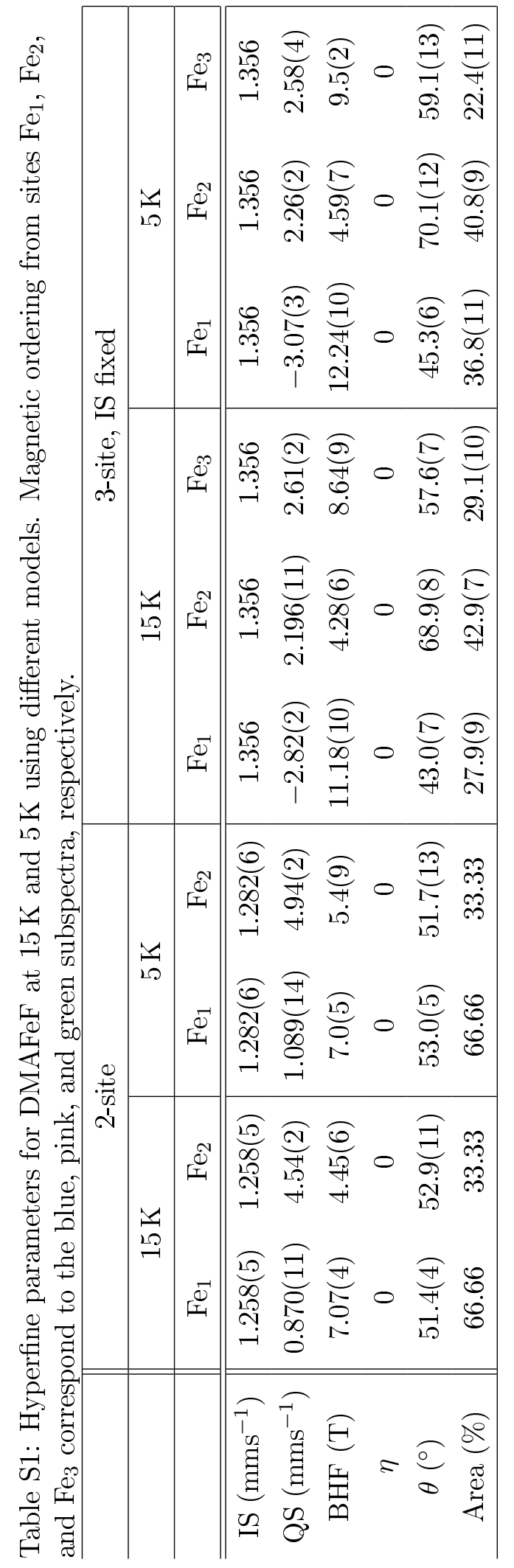




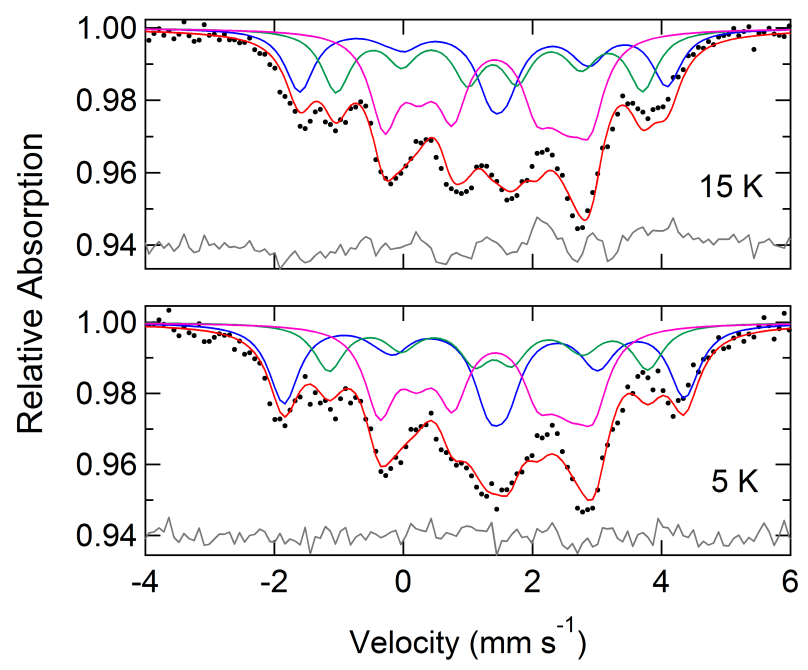

Figure S2: Magnetic sextet of DMAFeF at 15 and $5 \mathrm{~K}$ fitted with a three-site Fe model keeping the isomer shift fixed at $1.356 \mathrm{mms}^{-1}$. The peak widths are constrained to be the same. The black points indicate the collected data, the red line is the total fit to the spectrum, the blue, pink, and green lines are from the different Fe environments, and the grey line indicates the difference between the data and the overall fit. 


\section{Low-temperature SCXRD}

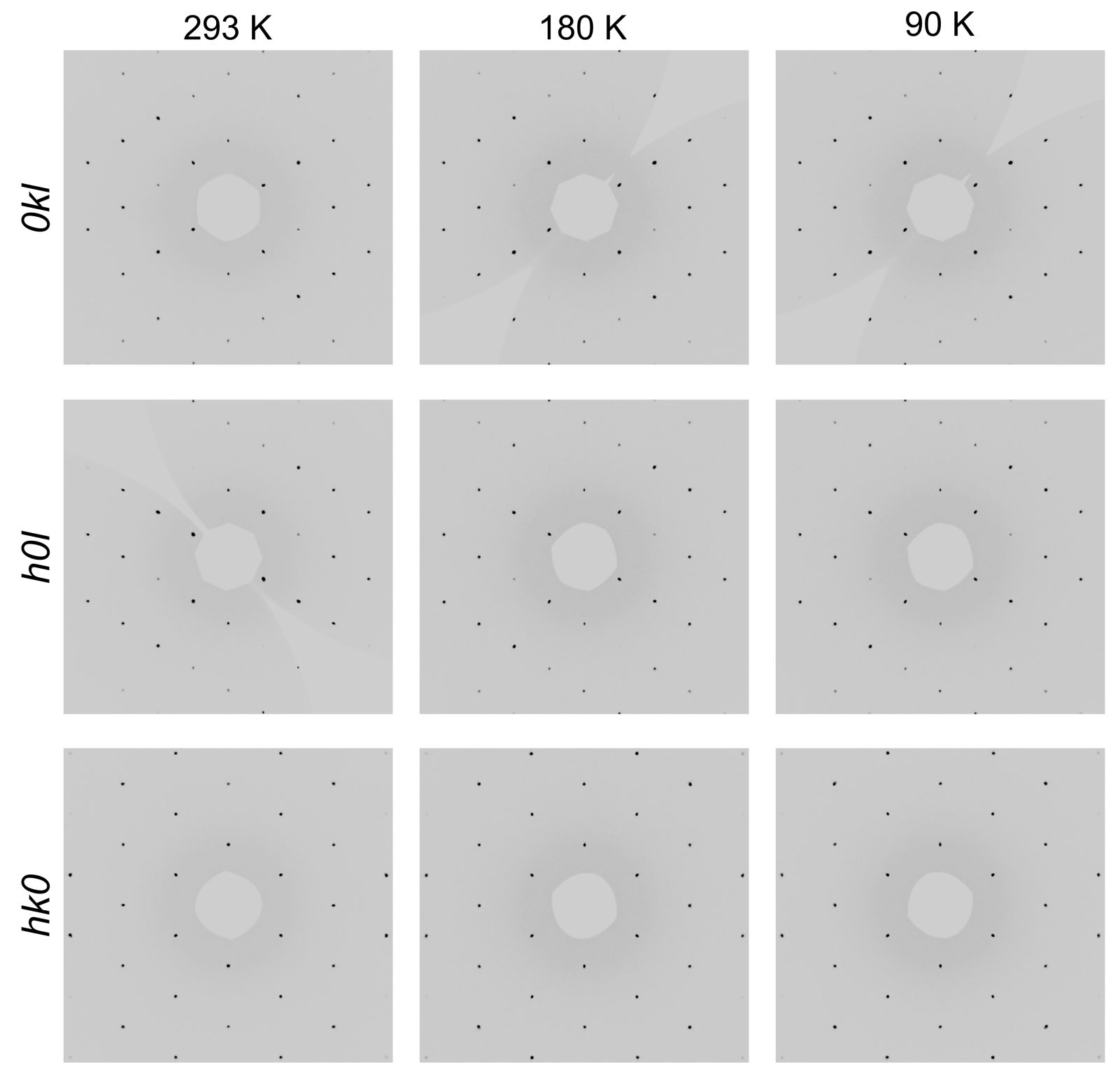

Figure S3: $0 k l, h 0 l$, and $h k 0$ reciprocal plane reconstructions for DMAFeF at ambient temperature and upon cooling to $180 \mathrm{~K}$ and $90 \mathrm{~K}$. 


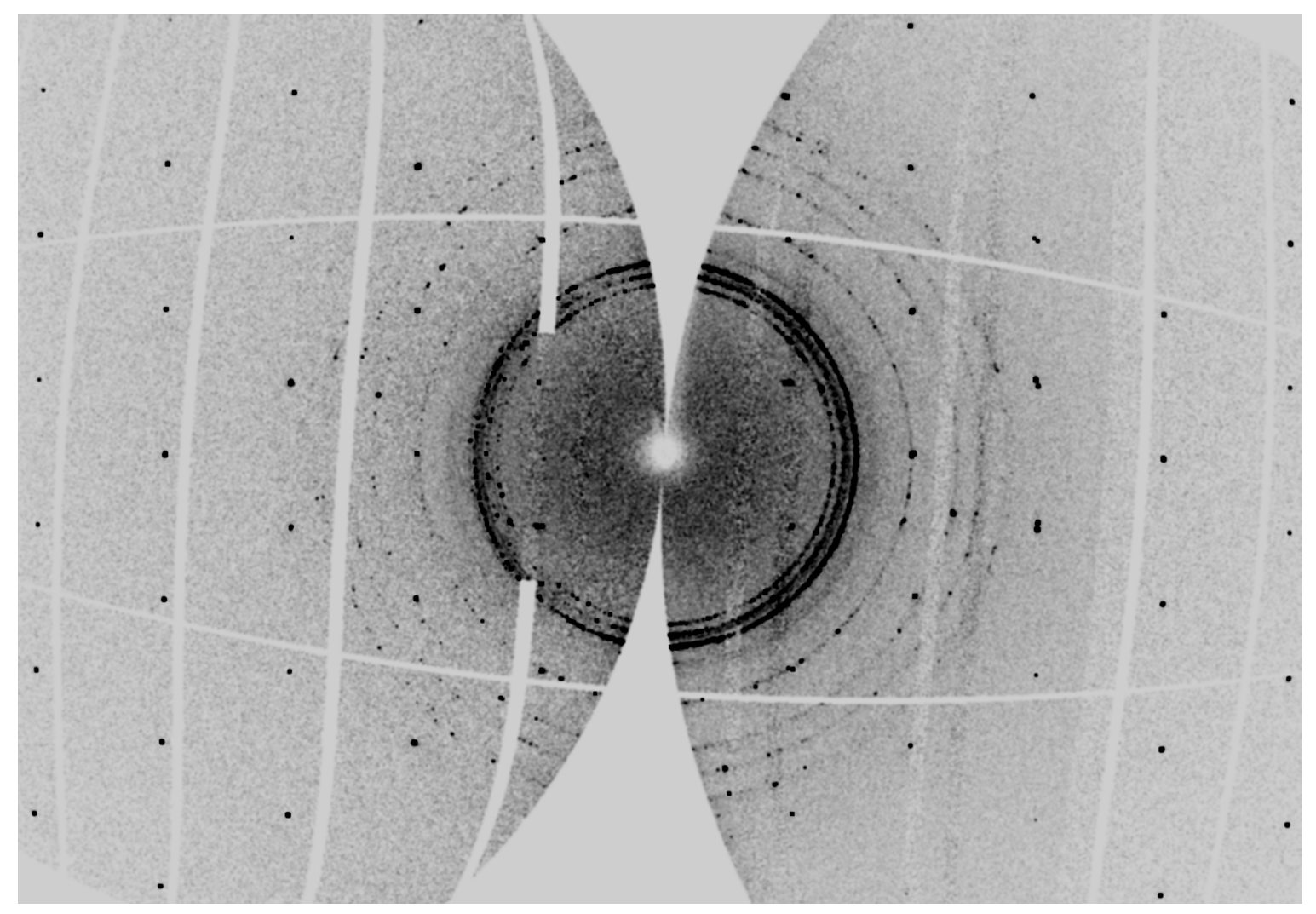

Figure S4: The $h k 0$ reciprocal plane reconstruction for DMAFeF at $4.2 \mathrm{~K}$. The rings of spots are from the ice formation. 
Table S2: Crystallographic details of DMAFeF at ambient, $180 \mathrm{~K}$ and $90 \mathrm{~K}$.

\begin{tabular}{|c|c|c|c|}
\hline & $293 \mathrm{~K}$ & $180 \mathrm{~K}$ & $90 \mathrm{~K}$ \\
\hline Formula & \multicolumn{3}{|c|}{$\mathrm{C}_{5} \mathrm{H}_{11} \mathrm{~N}_{1} \mathrm{O}_{6} \mathrm{Fe}_{1}$} \\
\hline$M_{\mathrm{w}}$ & \multicolumn{3}{|c|}{237} \\
\hline Crystal dimensions $(\mu \mathrm{m})$ & \multicolumn{3}{|c|}{$100 \times 100 \times 60$} \\
\hline Crystal System & trigonal & trigonal & trigonal \\
\hline Space Group & $R \overline{3} c$ & $R \overline{3} c$ & $R \overline{3} c$ \\
\hline Z & 6 & 6 & 6 \\
\hline$a(\AA)$ & $8.24495(14)$ & $8.23730(14)$ & $8.2357(2)$ \\
\hline$c(\AA)$ & $22.5145(4)$ & $22.3748(4)$ & $22.2928(7)$ \\
\hline$V\left(\AA^{3}\right)$ & $1325.47(5)$ & $1314.80(5)$ & $1309.47(9)$ \\
\hline \multicolumn{4}{|l|}{ Data collection } \\
\hline index ranges & $-11 \leq h \leq 11$ & $-11 \leq h \leq 11$ & $-11 \leq h \leq 11$ \\
\hline & $-11 \leq k \leq 11$ & $-11 \leq k \leq 11$ & $-11 \leq k \leq 11$ \\
\hline & $-31 \leq l \leq 31$ & $-31 \leq l \leq 31$ & $-30 \leq l \leq 30$ \\
\hline $\sin \left(\theta_{\max }\right) / \lambda\left(\AA^{-1}\right)$ & 0.697 & 0.697 & 0.698 \\
\hline \multicolumn{4}{|l|}{ No. of reflections } \\
\hline measured & 8323 & 8264 & 8194 \\
\hline unique & 428 & 427 & 424 \\
\hline unique with $\mathrm{I}>2 \sigma$ & 368 & 375 & 366 \\
\hline $\mathrm{R}_{\text {int }}$ & 0.0600 & 0.0652 & 0.0727 \\
\hline \multicolumn{4}{|l|}{ Refinement } \\
\hline No. of parameters & 34 & 34 & 34 \\
\hline No. of restraints & 3 & 3 & 3 \\
\hline$R_{1}[I>2 \sigma(I)]$ & 0.0227 & 0.0228 & 0.0281 \\
\hline$w R_{2}($ all data $)$ & 0.0503 & 0.0495 & 0.0620 \\
\hline Goodness-of-fit on $\mathrm{F}^{2}$ & 1.132 & 1.095 & 1.083 \\
\hline$\Delta \rho_{\max }, \Delta \rho_{\min }\left(e \AA^{-3}\right)$ & $0.26,-0.28$ & $0.31,-0.49$ & $0.48,-0.44$ \\
\hline
\end{tabular}




\section{HP-MS for AFeF}

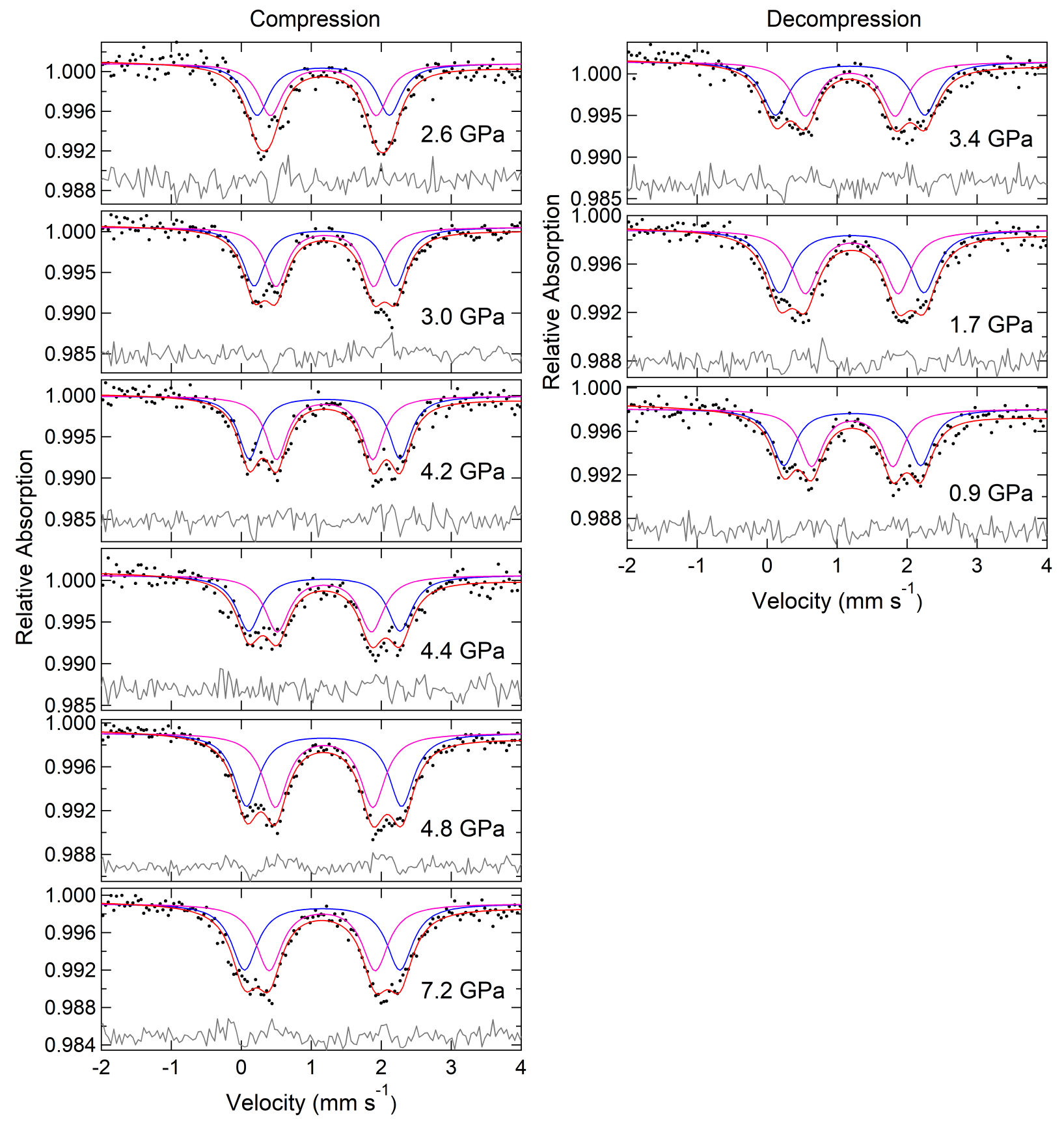

Figure S5: Mössbauer spectra collected for AFeF at variable pressure for compression and decompression. The black points indicate the collected data, the red line is the total fit to the spectrum, the blue and pink lines are from the Fe environments in $\mathrm{AFeF}$, and the grey line indicates the difference between the data and the fit. 


\section{HP-MS for DMAFeF}
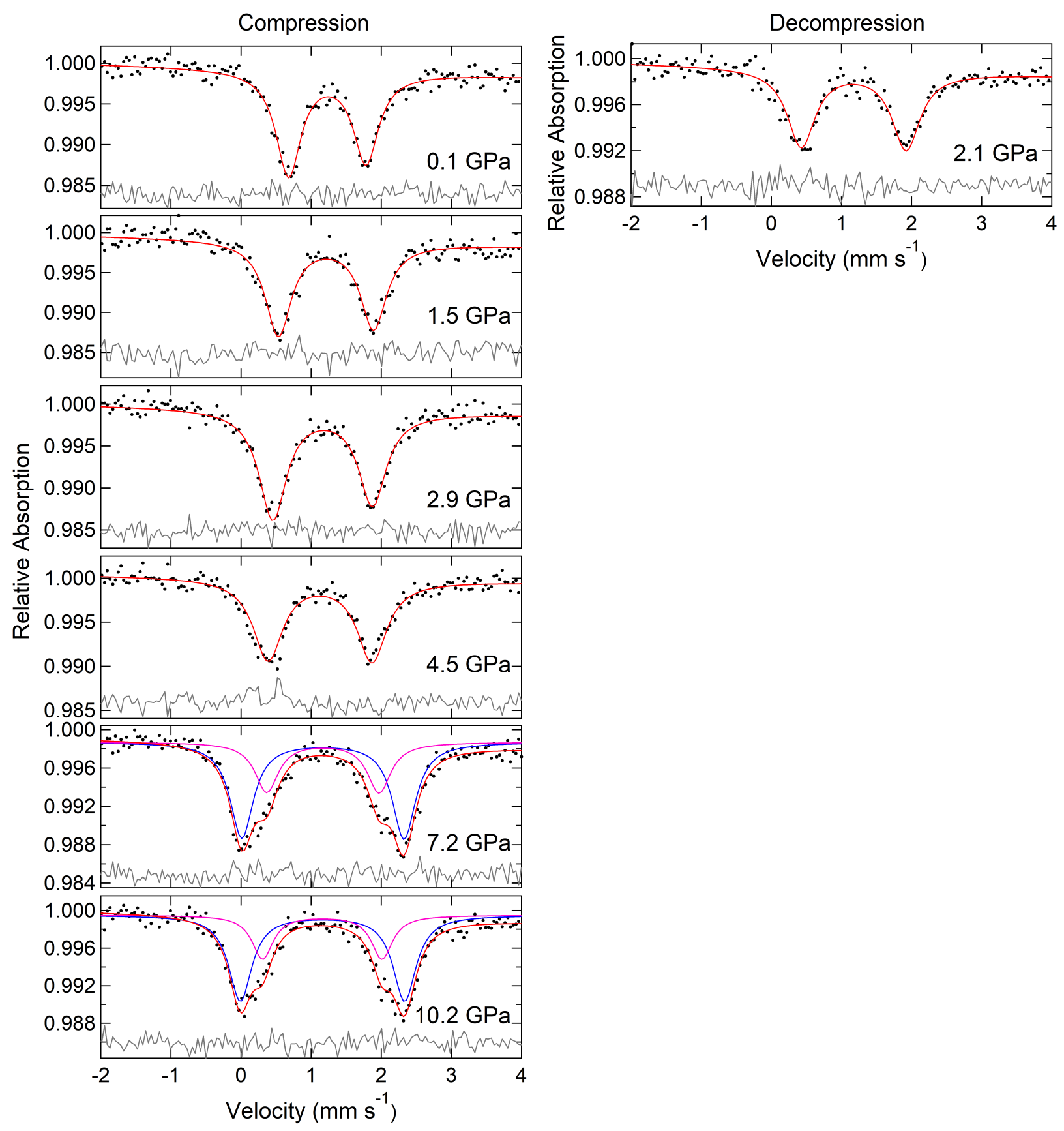

Figure S6: Mössbauer spectra collected for DMAFeF at variable pressure for compression and decompression. The black points indicate the collected data, the red line is the total fit to the spectrum, the blue and pink lines are from the Fe environments in DMAFeF, and the grey line indicates the difference between the data and the fit. 


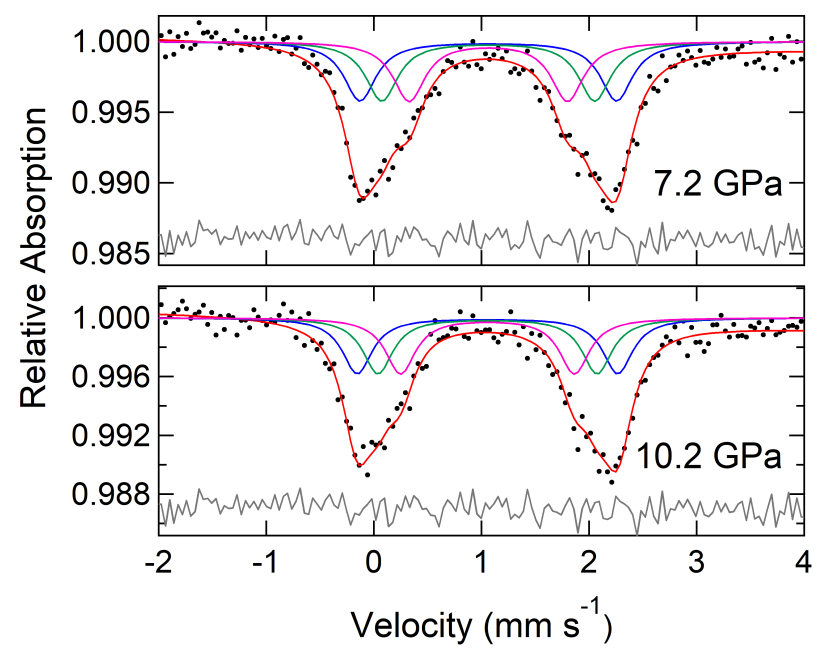

Figure S7: Mössbauer spectra of DMAFeF at 7.2 and 10.2 GPa fitted with a four-site Fe model. The IS, peak widths, and areas are constrained to be the same for all sites. Two of the iron sites exhibit very similar quadrupole splitting and thus lie nearly in the same position (blue spectrum). Therefore the QS was constrained to be the same for these two iron sites to allow reliable refinement of errors for the QS.

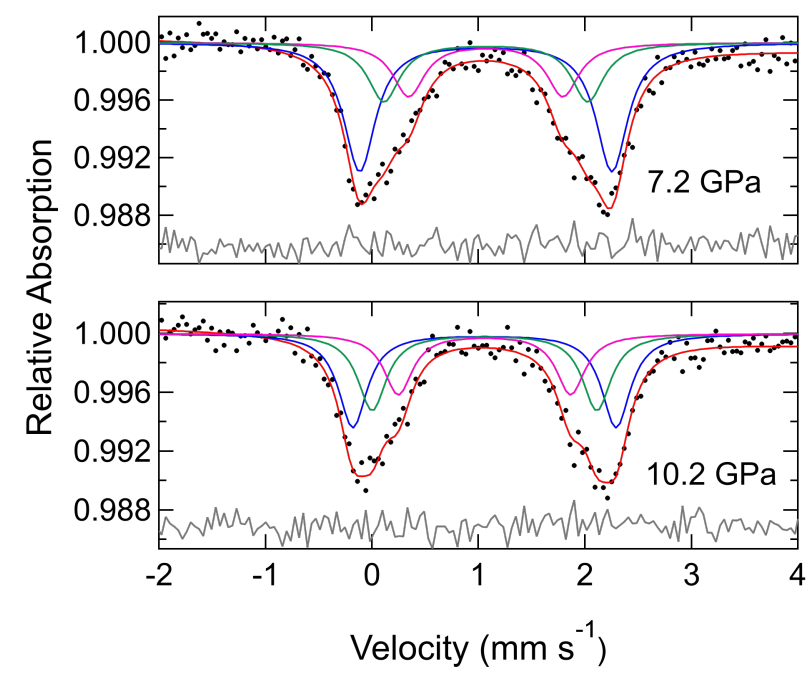

Figure S8: Mössbauer spectra of DMAFeF at 7.2 and 10.2 GPa fitted with a three-site Fe model. The IS and peak widths are constrained to be the same for all sites. 

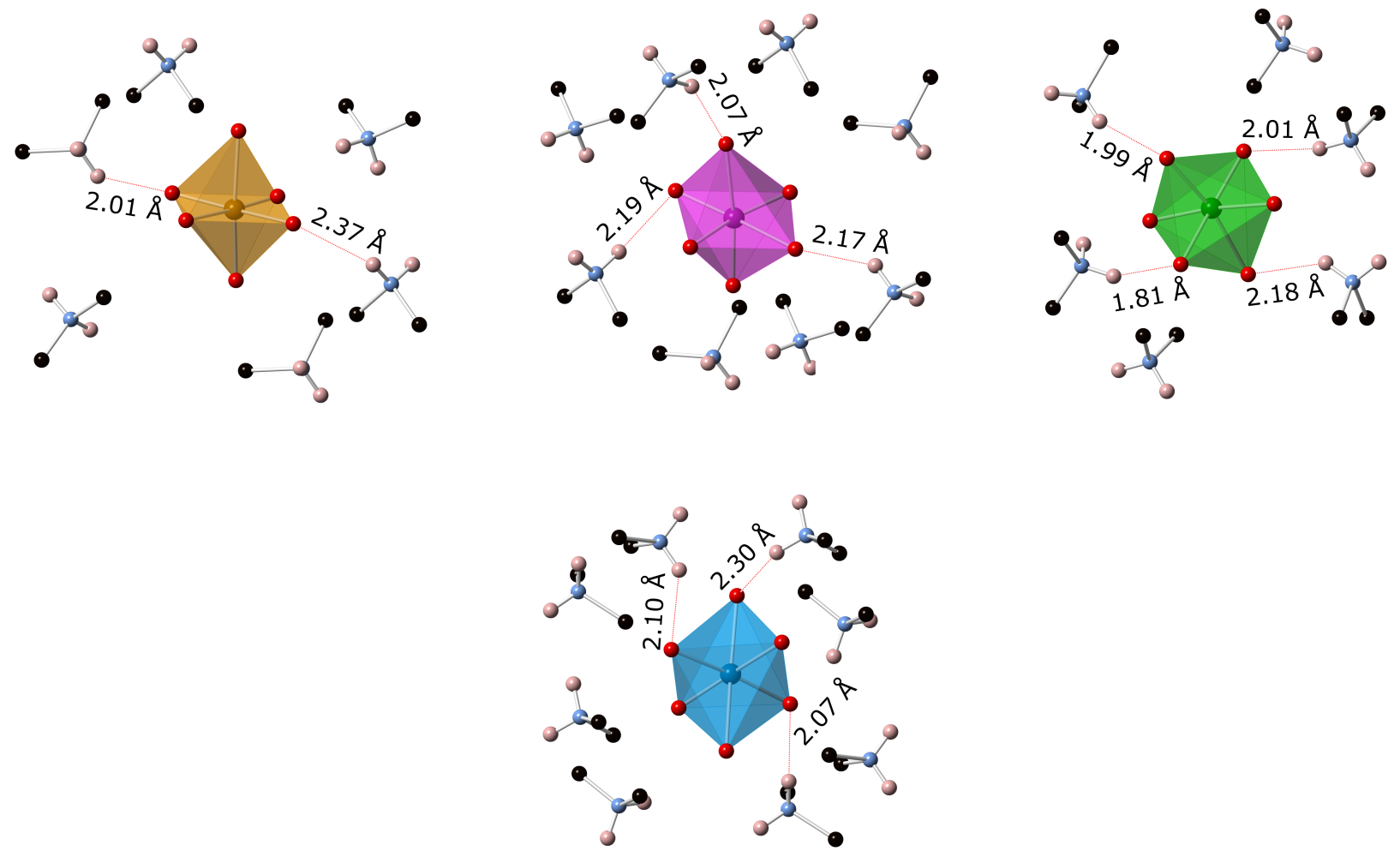

Figure S9: The four unique $\mathrm{FeO}_{6}$ octahedra in the high-pressure structure of DMAFeF at $7.9 \mathrm{GPa}$ from Ref. S 1. Hydrogen bonding interactions were placed for $\mathrm{H} \cdots \mathrm{O}$ distances below $2.4 \AA$ and $\mathrm{N}$... O distances below $3.0 \AA$. With these conditions, we observe three different H-bonding interactions to the $\mathrm{FeO}_{6}$ octahedra per unit cell: one with 2 hydrogen bonding interactions (25\%), one with 4 hydrogen bonding interactions (25\%), and two with 3 hydrogen bonding interactions $(50 \%)$. 


\section{LT- and HP-SMS for AFeF}
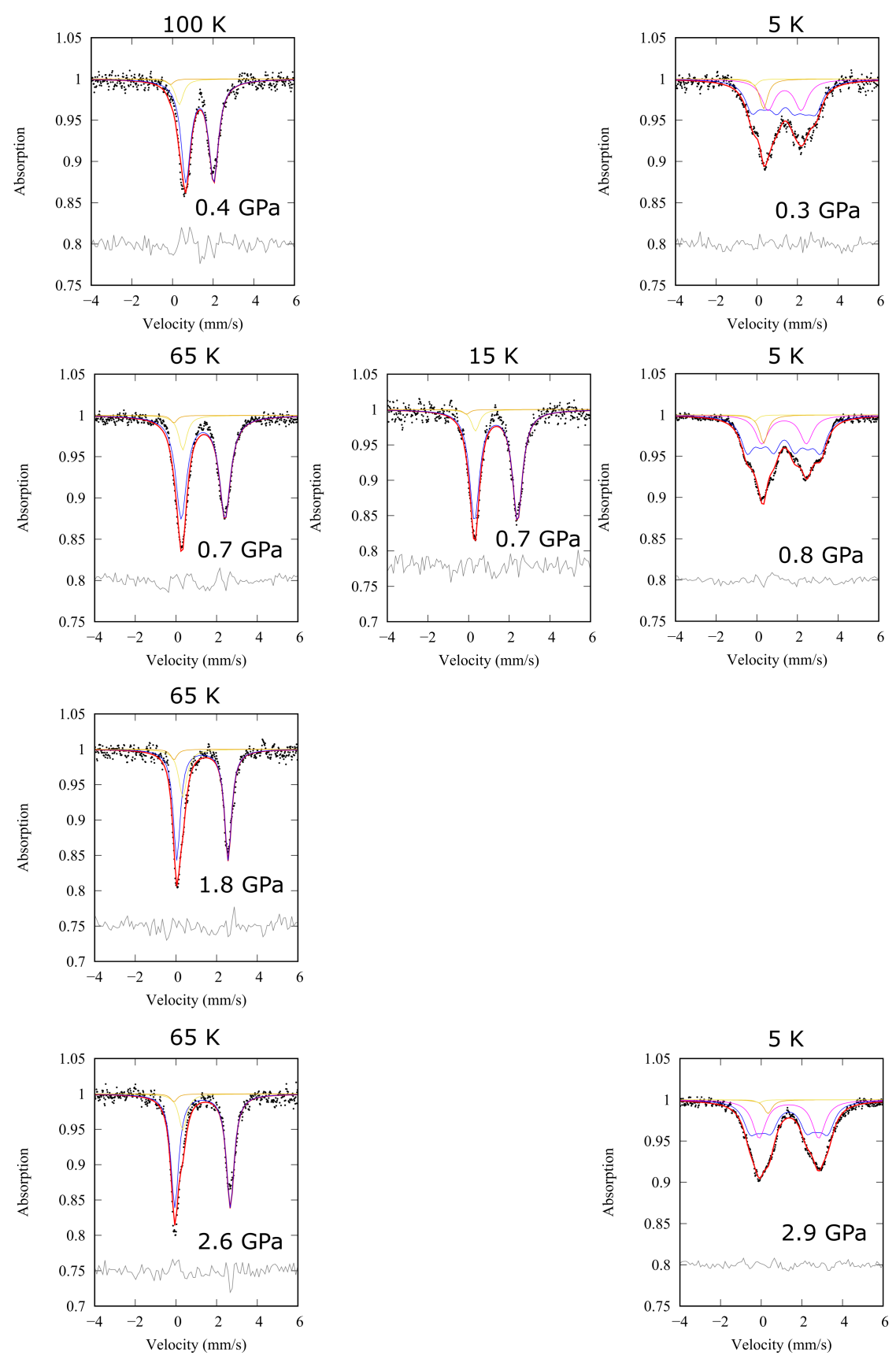

Figure S10: Selected SMS collected for AFeF at various temperature and pressure conditions. The black points indicate the collected data, the red line is the total fit to the spectrum, the blue and pink lines are from the Fe environments in $\mathrm{AFeF}$, the orange and yellow lines are the Fe contribution from the Be lens, and the grey line indicates the difference between the data and the fit. 

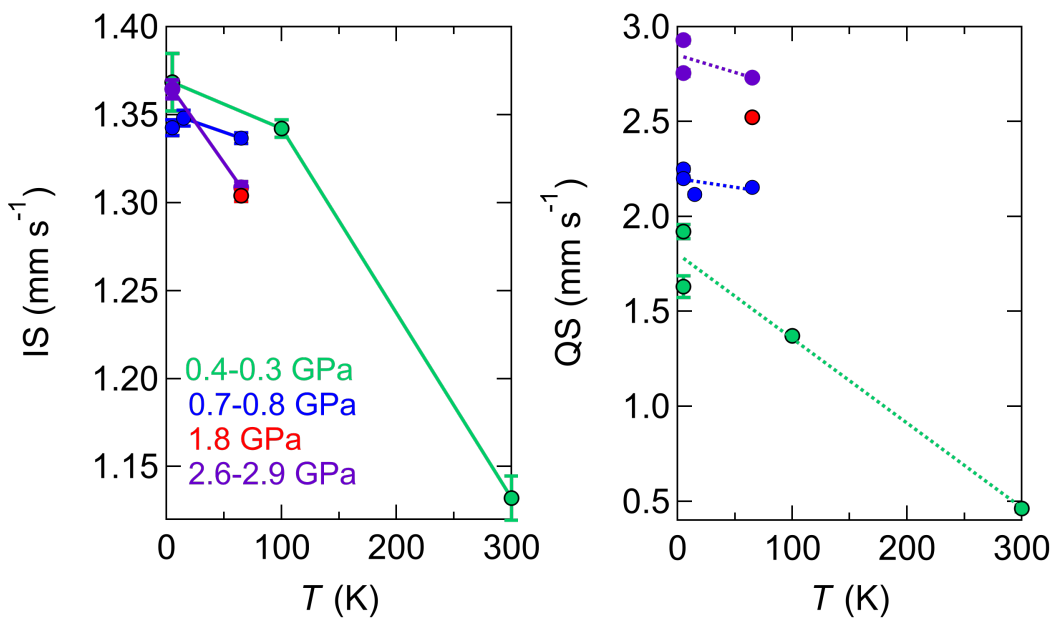

Figure S11: Temperature dependence of the isomer shift and quadrupole moment at different pressures for AFeF. Solid lines join up the markers, while the dotted lines indicate straight line fits. 


\section{LT- and HP-SMS for DMAFeF}
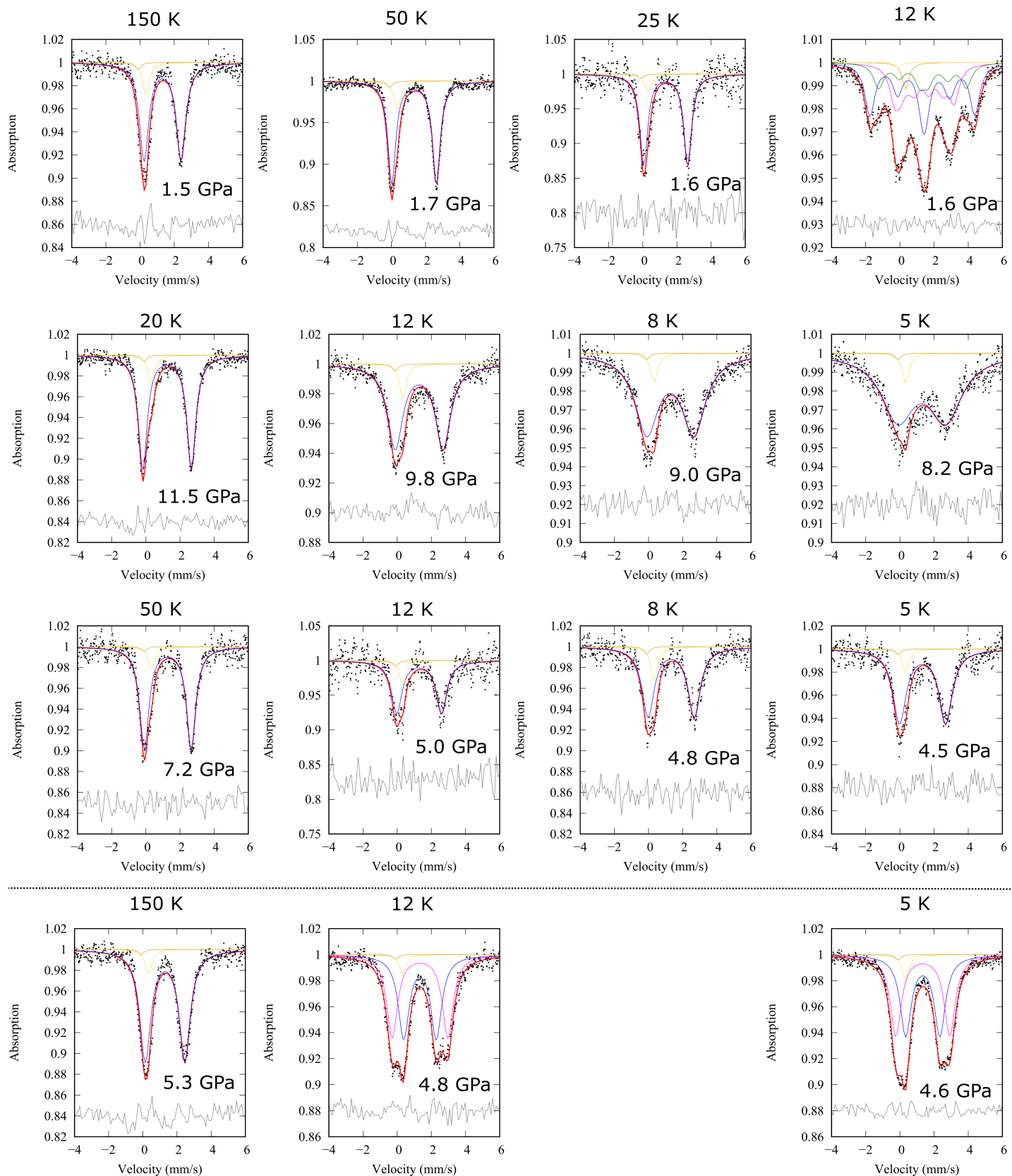

Figure S12: Selected SMS collected for DMAFeF at various temperature and pressure conditions, with each new line indicating a change in pressure (not arising from temperature changes). The dotted line indicates a new loading. The black points indicate the collected data, the red line is the total fit to the spectrum, the blue, pink, and green lines are from the Fe environments in DMAFeF, the orange and yellow lines are the Fe contribution from the Be lens, and the grey line indicates the difference between the data and the fit 

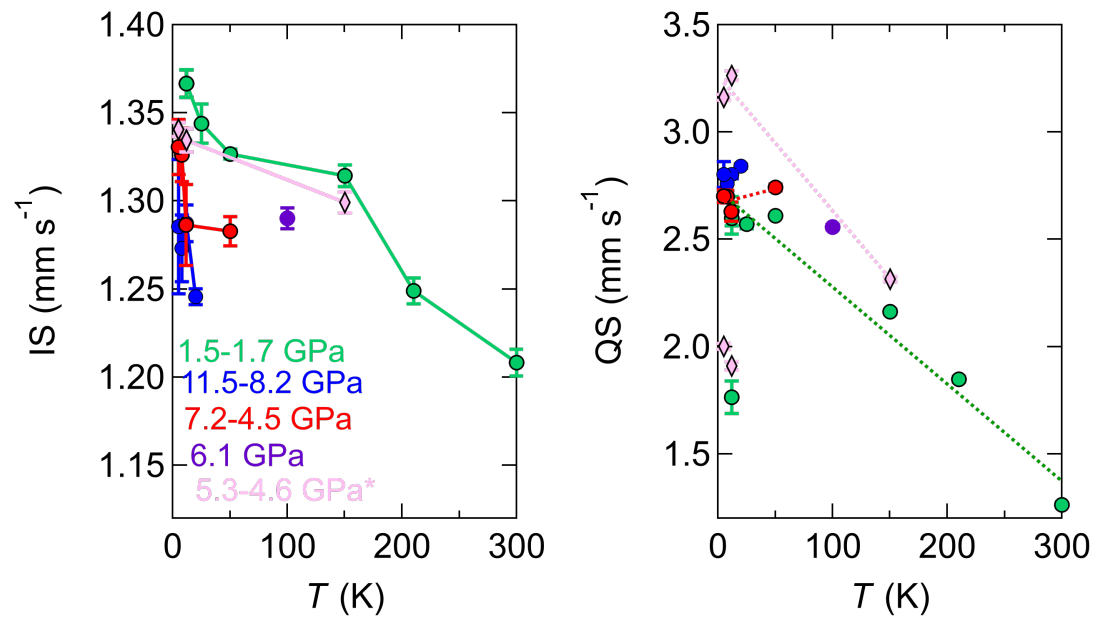

Figure S13: Temperature dependence of the isomer shift and quadrupole moment at different pressures for DMAFeF. The asterisk indicates the new loading (pink diamonds). Solid lines join up the markers, while the dotted lines indicate straight line fits (with only the high QS splitting used at LT). 


\section{References}

(S1) Collings, I. E.; Bykov, M.; Bykova, E.; Hanfland, M.; van Smaalen, S.; Dubrovinsky, L.; and Dubrovinskaia, N. Disorder-order transitions in the perovskite metal-organic frameworks $\left[\left(\mathrm{CH}_{3}\right)_{2} \mathrm{NH}_{2}\right]\left[\mathrm{M}(\mathrm{HCOO})_{3}\right]$ at high pressure. CrystEngComm 2018, 20, 3512-3521. 